\title{
Forum
}

\section{Challenges and opportunities for surveying and monitoring tropical biodiversity - a response to Danielsen et al.}

\author{
Jon Paul Rodríguez
}

Tropical conservation biologists face one of the biggest challenges of all time. Although the tropics harbour the majority of Earth's species, financial resources and trained personnel for conservation are lacking. While human populations in these regions attempt to increase their standards of living, their demands on ecosystem goods and services grow, just as in Europe and the United States centuries ago. Combined with weak institutions and ineffective law enforcement, these factors create processes that threaten the region's species and habitats and which become increasingly difficult to tackle. If we are to succeed in conserving tropical biodiversity we must act quickly and effectively.

A central problem is that the majority of tropical organisms remain unknown. Even among the betterknown groups new species continue to be described. For species that have already been described, there may be only limited knowledge of their distributions, no information on their relative abundances, and fewer data on their dynamics. Available data, such as those generated from specimens deposited in natural museums, are of limited use: sampling of tropical locations tends to be patchy, thus not adequately reflecting true patterns of the distribution of organisms. Under these circumstances it is nearly impossible to embark on systematic conservation planning, and there is no baseline for future monitoring efforts.

Recognizing the constraints imposed by working in tropical countries, Danielsen et al. (2003) propose an innovative method for biodiversity monitoring in protected areas in the Philippines. They consider the opportunities posed by the social and natural contexts, and demonstrate how such a programme may be carried out. They also recognize the limitations of their design and imply an adaptive approach for future adjustments.

The only weaknesses in Danielsen et al.'s proposal are, firstly, that it may not be readily replicable in other countries (as it is context-dependent) and, secondly, that it may not necessarily generate adequate data for quantitative monitoring. Danielsen et al. also indicate that changing donor priorities are a major threat to more

Jon Paul Rodríguez Centro de Ecología, Instituto Venezolano de Investigaciones Científicas, Apdo. 21827, Caracas 1020-A, Venezuela. E-mail: jonpaul@ivic.ve

Accepted 14 July 2003 elaborate monitoring schemes, and that they should thus be avoided in favour of "methods [that] are very simple, cost-effective and possible to sustain with limited external support." Although I agree with the spirit of this statement, it is important to keep in mind the role of funding agencies in the failure of monitoring efforts. The fact that " $[\mathrm{m}] \mathrm{any}$ programmes collapse immediately when the donor funding stops" is the responsibility of both the organization receiving the funds and the agency disbursing them. Donors must realize that the deficiencies in financial and human resources characteristic of tropical countries will not be solved without developing funding mechanisms to provide long-term support for both generating the necessary data for biodiversity management and building the capacity to see them through.

But the greatest challenge lies in the fact that the baseline data for monitoring biodiversity in tropical countries are not available. Large-scale systematic sampling and monitoring schemes (e.g. Margules \& Austin 1991) have yet to be implemented anywhere in the tropics, and this is where the recommendations of Yoccoz et al. (2001) are relevant. Careful design must be part of any biodiversity monitoring effort, not just those undertaken in developed countries (e.g. Rodríguez \& Sharpe, 2002). The fact that the challenge is greater in developing countries only means that we must be more intelligent, creative and effective in the use of limited resources.

\section{Acknowledgements}

I am grateful to Kathryn M. Rodríguez-Clark for her comments, and to Wildlife Trust for their support.

\section{References}

Danielsen, F., Mendoza, M.M., Alviola, P., Balette, D.S., Enghoff, M., Poulsen, M.K. \& Jensen, A.E. (2003) Biodiversity monitoring in developing countries: what are we trying to achieve? Oryx, 37, 407-409.

Margules, C.R. \& Austin, M.P. (eds) (1991) Nature Conservation: Cost Effective Biological Surveys and Data Analysis. Commonwealth Scientific and Industrial Research Organization, Canberra, Australia.

Rodríguez, J.P. \& Sharpe, C.J. (2002) NeoMaps: the Neotropical Biodiversity Mapping Initiative. Cotinga, 17, 13-14.

Yoccoz, N.G., Nichols, J.D. \& Boulinier T.(2001) Monitoring of biological diversity in space and time. Trends in Ecology and Evolution, 16, 446-453. 